\title{
Optimal Lipschitz extensions
}

\author{
Thanh Viet Phan $^{1, *}$ \\ ${ }^{1}$ Applied Analysis Research Group, Faculty of Mathematics and Statistics, Ton Duc Thang University, \\ Ho Chi Minh City, Vietnam
}

\begin{abstract}
The classical Lipschitz extension problem in concerned for conditions on a pair of metric spaces $\left(X, d_{X}\right)$ and $\left(Y, d_{Y}\right)$ such that for all $\Omega \subset X$ and for all Lipschitz function $f: \Omega \rightarrow Y$, then there is a function $g: X \rightarrow Y$ that extends $f$ and has the same Lipschitz constant as $f$. In this paper we discuss some results and open questions related to that issue.
\end{abstract}

\section{Introduction}

In this paper, we discuss the Lipschitz extension problems.

Let $\left(X, d_{X}\right)$ and $\left(Y, d_{Y}\right)$ be metric spaces and let $\Omega$ be a subset of $X$. Let $f: \Omega \rightarrow Y$ be a Lipschitz function. We denote

$$
\operatorname{Lip}(f, \Omega):=\sup _{\substack{x, y \in \Omega \\ x \neq y}} \frac{d_{Y}(f(x), f(y))}{d_{X}(x, y)}
$$

to be the Lipschitz constant of $f$ on $\Omega$.

Question 1.1 Whether there exists a function $g: X \rightarrow Y$ that extends $f$ and has the same Lipschitz constant as $f$, i.e. $\operatorname{Lip}(f, \Omega)=\operatorname{Lip}(g, X)$ ?

The pair $(X, Y)$ satisfies the property in Question 1.1 for all $\Omega \subset X$ and for all Lipschitz function $f: \Omega \rightarrow Y$ is said to have the isometric extension property. In Section 2 we present some famous examples for a pair of metric spaces $(X, Y)$ to have this property.

We consider a pair of metric space $(X, Y)$ that has isometric extension property. Let $\Omega$ be a subset of $X$ and let $f: \Omega \rightarrow Y$ be a Lipschitz function. If $g$ extends $f$ and $\operatorname{Lip}(g, X)=$ $\operatorname{Lip}(f, \Omega)$, then we say that $g$ is a minimal Lipschitz extension (MLE) of $f$. Of course, the MLE may not be unique, and it is possible to search for extension with additional properties. In a series of papers in the 1960's [2-4], Aronsson proposed the notion of an absolutely minimal Lipschitz extension (AMLE):

Definition 1.2 A function $u$ defined on $X$ is called an AMLE of $f$ if $u$ is a MLE of $f$ and $u$ satisfies

$$
\operatorname{Lip}(u, V)=\operatorname{Lip}(u, \partial V), \text { for any open } V \subset \subset X \backslash \Omega,
$$

where $\partial V$ denotes the boundary of $V$ and $V \subset \subset X$ means that $\bar{V} \subset X$.

\footnotetext{
*e-mail: phanthanhviet@tdtu.edu.vn
} 
Question 1.3 Whether there exists an AMLE $u: X \rightarrow Y$ of $f$ ? If this extension exists, is it unique?

After the works of Aronsson, there has been many studies devoted to the study of AMLEs. Although this problem was originated in real and functional analysis, it has been found relevant to many areas in applied mathematics (see $[16,23]$ for instance). In Section 3 we discuss the existence and uniqueness of AMLE, as well as problems related to them.

Now, given a function $f: \Omega \subset \mathbb{R}^{n} \rightarrow \mathbb{R}$. If $f$ is differentiable in some sense, can we extend it to a function on the whole $\mathbb{R}^{n}$ with the same differentiability? This question goes back to Whitney [29] in 1934. The problem is particularly interesting when $f$ is not smooth, and one looks for an extension satisfying certain constraints. Motivated by Taylor's expansion for smooth functions,

$$
f(y)=f(x)+\langle\nabla f(x), y-x\rangle+o(\|y-x\|),
$$

Whitney[29] and Glaeser[15] have introduced the notion of 1-fields for non-smooth functions: for $f: \Omega \rightarrow \mathbb{R}^{n}$ and $D f: \Omega \rightarrow \mathbb{R}^{n}$, we define the 1-field $F: \Omega \rightarrow \mathcal{P}^{1}\left(\mathbb{R}^{n}, \mathbb{R}\right)$, where

$$
\mathcal{P}^{1}\left(\mathbb{R}^{n}, \mathbb{R}\right):=\left\{P: \mathbb{R}^{n} \rightarrow \mathbb{R} \mid \exists p \in \mathbb{R}, v \in \mathbb{R}^{n}: P(a)=p+\langle v, a\rangle, \forall a \in \mathbb{R}^{n}\right\}
$$

(the set of first degree polynomials), by the identity

$$
F(x)(y):=f(x)+\langle D f(x), y-x\rangle, \quad \forall x \in \Omega, y \in \mathbb{R}^{n} .
$$

The 1-field $F$ can be interpreted as a smooth approximation for $f$ on $\Omega$, and hence the extension problem for $f$ can be related to the extension problem for $F$. Note that $F$ is welldefined even if $f$ and $D f$ has no relation as a priori.

In 2009, Le Gruyer [20] introduced the Lipschitz constant of 1-field $F$ :

$$
\Gamma^{1}(F ; \Omega):=2 \sup _{\substack{x, y \in \Omega \\ x \neq y}} \sup _{\substack{x \neq \mathbb{R}^{n}\\}} \frac{|F(x)(a)-F(y)(a)|}{\|x-a\|^{2}+\|y-a\|^{2}},
$$

Question 1.4 Does the Lipschitz constant $\Gamma^{1}$ of 1-field play a role in the extension problem similarly to the Lipschitz constant Lip of continuous function?

Question 1.4 and related issues to the 1-field extension are discussed in Section 4.

\section{Kirszbraun theorem and McShane-Whitney extremal extensions}

It is rare for a pair of metric spaces $(X, Y)$ to have the isometric extension property. In this section we present some famous examples for a pair of metric spaces $(X, Y)$ to have the isometric extension property.

\subsection{Kirszbraun theorem}

Kirszbraun in 1934 [18] proved that if $\mathrm{X}$ and $\mathrm{Y}$ are Euclidean spaces both equipped with the Euclidean norm then $(X, Y)$ have the isometric extension property. Later Frederick Valentine in 1943 [28] generalized it to pairs of Hilbert spaces of arbitrary dimension. This theorem is called Kirszbraun theorem, sometimes it is also called Kirszbraun-Valentine theorem. 
Theorem 2.1 (Kirszbraun theorem) Let $H_{1}$ and $H_{2}$ be Hilbert spaces. If $\Omega$ is a subset of $H_{1}$, and $f: \Omega \rightarrow H_{2}$ is a Lipschitz function, then there is a function $g: H_{1} \rightarrow H_{2}$ satisfying

$$
g=f \text { on } \Omega, \text { and } \operatorname{Lip}\left(g, H_{1}\right)=\operatorname{Lip}(f, \Omega) .
$$

The proof of the theorem uses geometric features of Hilbert spaces, the corresponding statement for Banach spaces is not true in general, not even for finite-dimensional Banach spaces. We can construct counterexamples where the domain is a subset of $\mathbb{R}^{n}$ with the maximum norm and the map is valued in $\mathbb{R}^{m}$ with Euclidean norm. A simple counterexample is the following:

$$
\begin{aligned}
& X=\mathbb{R}^{2} \text { with }\|x\|_{1}=\sup \left\{\left|x_{1}\right|,\left|x_{2}\right|\right\}, \\
& Y=\mathbb{R}^{2} \text { with }\|x\|_{2}=\left(x_{1}^{2}+x_{2}^{2}\right)^{1 / 2}, \\
& \Omega=\{(1,-1),(-1,1),(1,1)\} \subset X, \quad f: \Omega \rightarrow Y, \\
& f(1,-1)=(1,0), \quad f(-1,1)=(-1,0), \quad f(1,1)=(0, \sqrt{3}) .
\end{aligned}
$$

Then

$$
\|x-y\|_{1}=\|f(x)-f(y)\|_{2}=2, \quad \forall x, y \in \Omega .
$$

$\operatorname{Thus} \operatorname{Lip}(f, \Omega)=1$.

On the other hand, we have $\|x\|=1$ for all $x \in \Omega$, but there exists no $\xi \in \mathbb{R}^{2}$ such that

$$
\|f(x)-\xi\| \leq 1, \quad \forall x \in \Omega .
$$

Thus $f$ has no extension $u$ to $\Omega \cup\{(0,0)\}$ with $\operatorname{Lip}(u, \Omega \cup\{(0,0)\})=1$.

More generally, the theorem fails for $\mathbb{R}^{m}$ equipped with any $\ell_{p}$ norm $(p \neq 2)$ (see Schwartz 1969 [26, p. 20]).

It is easy to see that the Kirszbraun theorem is equivalent to the following theorem.

Theorem 2.2 [8, Proposition 1.13] Let $H_{1}$ and $H_{2}$ be Hilbert spaces, $\left\{x_{\beta}\right\}_{\beta \in I}$ a subset of $H_{1}$, $\left\{y_{\beta}\right\}_{\beta \in I}$ a subset of $\mathrm{H}_{2}$, and $\left\{r_{\beta}\right\}_{\beta \in I}$ a collection of nonnegative real numbers. If

$$
\left\|y_{\beta}-y_{\gamma}\right\| \leq\left\|x_{\beta}-x_{\gamma}\right\|
$$

for all $\beta, \gamma \in I$, then $\bigcap_{\beta \in I} B\left(y_{\beta}, r_{\beta}\right) \neq \emptyset$ whenever $\bigcap_{\beta \in I} B\left(x_{\beta}, r_{\beta}\right) \neq \emptyset$.

If $H_{1}=H_{2}$ then the contraction hypothesis (3) in Theorem 2.2 implies that we can rearranging the ball $B\left(x_{\beta}, r_{\beta}\right)$ in a way that the centers of the balls are closer to each other. In addition, if $\operatorname{dim} H_{1}=\operatorname{dim} H_{2}<+\infty$ and the index $I$ is finite, then it is natural to conjecture that the volume of the intersection should increase as we push the spheres together. In fact, we have

Problem 2.3 [19, 24, Kneser-Poulsen conjecture] Let $\left\{r_{1}, \ldots, r_{N}\right\}$ be a collection of nonnegative numbers and $\left\{x_{1}, \ldots, x_{N}\right\}$ and $\left\{y_{1}, \ldots, y_{N}\right\}$ two subsets of $\mathbb{R}^{n}$. If $\left\{y_{1}, \ldots, y_{N}\right\}$ is a contraction of $\left\{x_{1}, \ldots, x_{N}\right\}$, i.e

$$
\left\|y_{i}-y_{j}\right\| \leq\left\|x_{i}-x_{j}\right\|,
$$

for all $1 \leq i, j \leq N$. Then

$$
\operatorname{Vol}\left(\bigcap_{i=1}^{N} B\left(y_{i}, r_{i}\right)\right) \geq \operatorname{Vol}\left(\bigcap_{i=1}^{N} B\left(x_{i}, r_{i}\right)\right)
$$

The above was conjectured independently by Kneser [19] in 1955 and Poulsen [24] in 1954. It is quite surprising that the conjecture remains unproven for a long time. The conjecture is solved only when $n=2$ by Bezdek and Connelly [9] in 2002 and it is still open when $n \geq 3$. 


\subsection{McShane-Whitney extremal extensions}

McShane [22] and Whitney [29] in 1934 proved that if $Y=\mathbb{R}$ and for any arbitrary metric space $X$ then $(X, Y)$ have the isometric extension property. More precisely, they prove that for any metric space $X$ and any $\Omega$ subset of X, every Lipschitz function $f: \Omega \rightarrow \mathbb{R}$ has two MLEs which can be written explicitly :

$$
\begin{aligned}
& m^{+}(\xi)=\inf _{x \in \Omega}\left\{f(x)+\operatorname{Lip}(f, \Omega) d_{X}(x, \xi)\right\} \text { for } \xi \in X, \\
& m^{-}(\xi)=\sup _{x \in \Omega}\left\{f(x)-\operatorname{Lip}(f, \Omega) d_{X}(x, \xi)\right\} \text { for } \xi \in X .
\end{aligned}
$$

Moreover, $m^{ \pm}$are extremal: the first is maximal and the second is minimal, that is

$$
m^{-}(x) \leq g(x) \leq m^{+}(x), \forall x \in X,
$$

for any $g$ other MLE of $f$.

Clearly MLEs are unique if and only if $m^{+}=m^{-}$on $X$. This rarely happens.

\section{Absolutely minimal Lipschitz extension}

We consider the case $X \subset \mathbb{R}^{n}$ and $Y=\mathbb{R}$ both equipped with the Euclidean norm. Let $\Omega=\partial X$ and $f: \Omega \rightarrow \mathbb{R}$. From McShane - Whitney formulas (4) and (5), we have two extremal MLEs $m^{+}$and $m^{-}$of $f$. Thus, unless $m^{+} \equiv m^{-}$, we have no uniqueness of MLE of $f$. In this section we discuss about the question of the existence and uniqueness of AMLE of $f$.

\subsection{The existence and uniqueness of AMLE}

We rewrite the original problem in (1) by calculus of variations problems in the sup-norm:

$$
\|\nabla u\|_{L^{\infty}(V)} \leq\|\nabla v\|_{L^{\infty}(V)},
$$

for all $V \subset X$, and for all "sufficiently regular" $v$ satisfying $v=u$ on $\partial V$.

It is not clear at all that (6) is equivalent to (1). If $V$ is convex then $\operatorname{Lip}(v, V)=\|\nabla v\|_{L^{\infty}(V)}$ for any locally Lipschitz continuous function $v$. But if $V$ is not convex, then $\operatorname{Lip}(v, V)$ may be infinite when $\|\nabla v\|_{L^{\infty}(V)}=1$. The equivalence, which may not be generally appreciated, is established in [7].

A central question has been to understand minimization problems involving (6) and related functionals, and especially to develop appropriate PDE methods. The most popular line of research has arisen from the idea of interpreting the AMLE as a formal limit of $u_{p}$, as $p \rightarrow \infty$, where $u_{p}$ is the minimizing of the functional

$$
I_{p}[v]:=\int_{X}\|\nabla v\|^{p} d x
$$

where $X \subset \mathbb{R}^{n}$ is open, $v \in W^{1, p}(X ; \mathbb{R})$ with boundary condition $v=f$ on $\partial X$, and $\nabla v=$ $\left(u_{x_{1}}, \ldots, u_{x_{n}}\right)$ is the gradient of $v$.

The problem (7) leads as usual to the Euler-Lagrange equation $\Delta_{p} u=0$, but it was unclear what is the correct "Euler-Lagrange" equation for the problem (6). In 1967, Aronsson [4] discovered the "Euler-Lagrange" equation for the problem (6) by approximation procedure. Let us explain the idea at least formally. He considered for finite $p$ the nonlinear $p$-Laplacian operator:

$$
\Delta_{p} u=\operatorname{div}\left(\|\nabla u\|^{p-2} \nabla u\right)=(p-2)\|\nabla u\|^{p-4} \Delta_{\infty} u+\|\nabla u\|^{p-2} \Delta u
$$


where

$$
\Delta_{\infty} u:=\sum_{j, k=1}^{n} u_{x_{j}} u_{x_{k}} u_{x_{j} x_{k}} .
$$

Dividing the equation $\Delta_{p} u=0$ by $(p-2)\|\nabla u\|^{p-4}$ leads to

$$
\Delta_{\infty} u+\frac{1}{p-2}\|\nabla u\|^{2} \Delta u=0
$$

Let $p$ tend to infinity. This leads to the equation

$$
\Delta_{\infty} u=0
$$

where

$$
\Delta_{\infty} u:=\sum_{j, k=1}^{n} u_{x_{j}} u_{x_{k}} u_{x_{j} x_{k}} .
$$

This nonlinear equation is a highly degenerate elliptic equation. The above "EulerLagrange" equation is nowadays known as the infinity Laplace equation.

In 1967, Aronsson [4] proved that if $X$ is the closure of the bounded domain in $\mathbb{R}^{n}$ and $f$ is a Lipschitz function given on $\partial X$ then a $C^{2}$ function $u: X \rightarrow \mathbb{R}$ is an AMLE of $f$ if and only if it is a solution of the infinity Laplace equation (8) with boundary condition $u=f$ on $\partial X$.

However, AMLEs are not $C^{2}$ in general, the classical solutions of the eikonal equation $|D u|=$ constant is an example of AMLEs which are not $C^{2}$. The existence and uniqueness of AMLEs was unknown in general, and it was also unclear what is correct notion for nonsmooth solution of infinity Laplace equation (8). An important breakthrough in this direction was made by Jensen by using the viscosity solution concept:

Definition 3.1 (i) An upper semi-continuous function $u: \bar{X} \rightarrow \mathbb{R}$ is a sub-solution of (8) if $\Delta_{\infty} \phi(x) \geq 0$, for every $(x, \phi) \in X \times C^{2}(X)$ such that $(u-\phi)(x) \geq(u-\phi)(y)$ for all $y \in X$.

(ii) An lower semi-continuous function $u: \bar{X} \rightarrow \mathbb{R}$ is a super-solution of $(8)$ if $\Delta_{\infty} \phi(x) \leq 0$, for every $(x, \phi) \in X \times C^{2}(\Omega)$ such that $(u-\phi)(x) \leq(u-\phi)(y)$ for all $y \in X$.

(iii) A continuous function $u: \bar{X} \rightarrow \mathbb{R}$ is a viscosity solution of (8) if it is both a subsolution and super-solution.

This concept introduced by Crandall and Lions in a famous paper in 1983 [12] is a generalization of the classical concept to treat the problem for non-smooth function. The modern theory of viscosity solutions was developed by Crandall, Evans, Jensen, Ishii, Lions, and others. First, it was designed for first order equations. Later, it was extended to second order equations. The solutions must obey a Comparison Principle.

In 1993, Jensen [16] proved the existence and uniqueness of viscosity solution of infinity Laplace equation with boundary condition $u=f$ on $\partial X$. Jensen showed as well that this solution is also identified by Aronsson: for every open bounded subset $V$ of $X$ if $u$ is the viscosity solution of infinity Laplace equation then

$$
\|\nabla u\|_{L^{\infty}(V)} \leq\|\nabla v\|_{L^{\infty}(V)},
$$

for each $v \in C(\bar{V})$ and $u=v$ on $\partial V$.

After the foundational papers of Aronsson, the uniqueness of AMLE remained as the most important open problem. Jensen's work generated considerable interest in the long developments in the existence and uniqueness theory of AMLEs. 


\subsection{Comparison with cones and generalizing the AMLE in metric spaces}

The existence and uniqueness theory of AMLEs was settled in Jensen's influential work. Jensen's proof was rather indirect. Moreover, the arguments relied on the theory of variational problems and the viscosity solution machinery developed for second-order elliptic equations. A more direct uniqueness proof was found by Armstrong and Smart in 2010 [1] by using the comparison with cones concept:

Definition 3.2 ( $i$ ) A function $u \in C(X)$ is said to enjoys comparison with cones from above in $X$ if for every bounded open subset $V$ of $X$ and every $x_{0} \in \mathbb{R}^{n}, a, b \in \mathbb{R}$ for which

$$
u(x) \leq C(x)=a+b\left\|x-x_{0}\right\|
$$

holds on $\partial\left(V \backslash\left\{x_{0}\right\}\right)$, we have $u \leq C$ in $V$ as well.

(ii) A function $u \in C(X)$ is said to enjoys comparison with cones from below in $X$ if for every bounded open subset $V$ of $X$ and every $x_{0} \in \mathbb{R}^{n}, a, b \in \mathbb{R}$ for which

$$
u(x) \geq C(x)=a+b\left\|x-x_{0}\right\|
$$

holds on $\partial\left(V \backslash\left\{x_{0}\right\}\right)$, we have $u \geq C$ in $V$ as well.

(iii) A function $u \in C(X)$ is said to enjoys comparison with cones in $X$ if it enjoin comparison with cones both above and below.

Notice that any solution of an eikonal equation $|D v|=$ constant is a classical solution of $-\Delta_{\infty} v=0$ wherever it is smooth. Thus the cone $C(x)=a+b\left\|x-x_{0}\right\|$ is a classical solution for $x \neq x_{0}$.

In 2001, Crandall, Evans, and Gariepy [11] proved that if $u$ is a continuous function on a bounded open set $X \subset \mathbb{R}^{n}$ then the concepts of AMLE, the viscosity solution of infinity Laplace equation and the function enjoying comparison with cones are equivalent.

In 2010, Armstrong and Smart [1] presented an elegant and elementary proof of the uniqueness of the functions enjoying comparison with cones. This proof makes no use of partial differential equations and does not need the viscosity solution machinery developed for second-order elliptic equations. This implies a new and easy proof for the uniqueness of AMLE and the uniqueness of the viscosity solution of the infinity Laplace equation.

The definition of comparison with cones easily extends to other metric spaces. In 2007, Champion and De Pascale [10] adapted this definition to length spaces.

In 2002, Juutinen [17] used Perron's method to establish the existence of AMLE extensions for separable length space domains.

In 2009, Peres, Schramm, Sheffield and Wilson [23] used game-theoretic techniques to prove the existence and uniqueness of AMLE for general length spaces ${ }^{1}$. It relied on some complicated probabilistic arguments and a beautiful connection between the infinity Laplace equation and random-turn "tug of war" game.

Extending results on AMLEs to vector valued functions presents many difficulties, which in turn has limited the number of results in this direction, see e.g. [14, 27].

Problem 3.3 For $X=\mathbb{R}^{n}$ and $Y=\mathbb{R}^{m}(m \geq 2)$ both equipped with the Euclidean norm, is it true that for every $\Omega \subset X$, any Lipschitz function $f: \Omega \rightarrow Y$ admits an AMLE $g: X \rightarrow Y$ ?

\footnotetext{
${ }^{1} \mathrm{~A}$ metric space $\left(X, d_{X}\right)$ is a length space if for all $x, y \in X$, the distance $d_{X}(x, y)$ is the infimum of the lengths of curves in $X$ that connect $x$ to $y$.
} 


\subsection{Regularity}

We study the differentiability properties of viscosity solutions of the PDE

$$
\Delta_{\infty} u=0, \quad \text { in } X
$$

where $X \subset \mathbb{R}^{n}$ is an open set.

A viscosity solution $u$ of (10) is called an infinity harmonic function.

Example 3.4 The best known infinity harmonic function was exhibited by Aronsson in 1984 [5]: $u(x, y)=x^{4 / 3}-y^{4 / 3}$. The first derivatives of $u$ are Holder continuous with exponent $\frac{1}{3}$; the second derivatives do not exist on the lines $x=0$ and $y=0$. Therefore, $u \in C^{1}$ but $u \notin C^{2}$.

Problem 3.5 Are infinity harmonic functions necessarily continuously differentiable?

In 2011, Evans and Smart[13] established that the infinity harmonic function is everywhere differentiable. In 2006, Savin [25] has shown that infinity harmonic functions in $n=2$ variables are in fact continuously differentiable. Savin's arguments uses the topology of $\mathbb{R}^{2}$ very strongly, and it is difficult to general Savin's arguments for case $n>2$. The question of whether infinity harmonic functions are necessarily $C^{1}$ in general remains the most conspicuous open problem in the area.

Problem 3.6 Does an infinity harmonic function belong to $C_{l o c}^{1, \frac{1}{3}}$ ?

Savin [25] proved that in the plane all infinity harmonic functions have continuous gradients - Moreover from Example (3.4), the first derivatives of $u(x, y)=x^{4 / 3}-y^{4 / 3}$ are Holder continuous with exponent $\frac{1}{3}$. Thus we can hope that the optimal regularity class is $C_{l o c}^{1, \frac{1}{3}}$.

\section{$4 C^{1,1}$ extension}

In this Section, let $\Omega$ be a subset of the Euclidean space $\mathbb{R}^{n}$ and $F$ be a 1-field on $\Omega$ such that $f: \Omega \mapsto \mathbb{R}, D f: \Omega \mapsto \mathbb{R}^{n}$ are mappings associated with $F$.

\subsection{The minimal Lipschitz extension for 1 -field}

Let us review the Kirszbraun extension theorem (see Theorem 2.1): Let $\Omega \subset \mathbb{R}^{n}$ and let $u: \Omega \rightarrow \mathbb{R}^{m}$ be a Lipschitz function. Then there exists a total Lipschitz extension $v$ of $u$ such that $\operatorname{Lip}\left(v, \mathbb{R}^{n}\right)=\operatorname{Lip}(u, \Omega)$. As a consequence, we have $\operatorname{Lip}^{*}(u)=\operatorname{Lip}(u, \Omega)$ where

$$
\operatorname{Lip}^{*}(u):=\inf \left\{\operatorname{Lip}\left(v, \mathbb{R}^{n}\right): v \text { total extension of } u\right\} .
$$

The natural question is that what is

$$
\operatorname{Lip}^{*}(F)=\inf \left\{\operatorname{Lip}\left(D g, \mathbb{R}^{n}\right): G \text { is an extension of } F\right\} ?
$$

In 2009, Le Gruyer [20] proved that $\operatorname{Lip}^{*}(F)=\Gamma^{1}(F, \operatorname{dom}(F))$. Moreover, the constant $\Gamma^{1}$ of 1-field plays a role in the extension problem similarly to the constant Lip of continuous function: 
Theorem 4.1 [20] Let $F$ be a l-field. The functional $\Gamma^{1}: F \rightarrow \Gamma^{1}(F, \operatorname{dom}(F)) \in \mathbb{R}^{+} \cup\{+\infty\}$ is the unique one satisfying

$(P 0) \Gamma^{1}$ is increasing, that is, $G$ extends $F$ implies that

$$
\Gamma^{1}(G, \operatorname{dom}(G)) \geq \Gamma^{1}(F, \operatorname{dom}(F)) .
$$

(P1) If $G$ has total domain satisfying $\Gamma^{1}(G, \operatorname{dom}(G))<+\infty$, then the total function $g$ defined by $g(x):=G(x)(x)$ is differentiable and its derivative $\nabla g$ is Lipschitz.

(P2) If $g$ is a differentiable function of total domain with $\nabla g$ Lipschitz, then

$$
\Gamma^{1}\left(G, \mathbb{R}^{n}\right)=\operatorname{Lip}\left(\nabla g, \mathbb{R}^{n}\right)
$$

where $G$ is the 1-field associate to $g$, i.e. $G(x)(a):=g(x)+\langle\nabla g(x) ; a-x\rangle, \forall x, a \in \mathbb{R}^{n}$.

(P3) For any $F$ such that $\Gamma^{1}(F, \operatorname{dom}(F))<+\infty, F$ extends to a total 1-field $G$ satisfying

$$
\Gamma^{1}(G, \operatorname{dom}(G))=\Gamma^{1}(F, \operatorname{dom}(F)) .
$$

This theorem holds not only in $\mathbb{R}^{n}$ but in fact in any Hilbert space. Therefore, it generalizes the $C^{1,1}$ version of the classical Whitney extension theorem [29].

To compute the norm $\Gamma^{m}$ of the minimal extension on $C^{m}$ which generalize Le Gruyer's work on minimal $C^{1}$ extensions is a very difficult problem and the main thrust is some attempts to guess the natural norm for which one can obtain the minimal extension.

Problem 4.2 Let $\Omega$ be a subset of Euclidean space $\mathbb{R}^{n}$. Let $\mathcal{P}^{m}\left(\mathbb{R}^{n}, \mathbb{R}\right)$ be the set of $m$-degree polynomials mapping $\mathbb{R}^{n}$ to $\mathbb{R}$. Let us consider a m-field $T: \Omega \rightarrow \mathcal{P}^{m}\left(\mathbb{R}^{n}, \mathbb{R}\right)$. Find a functional $\Gamma^{m}: T \rightarrow \Gamma^{m}(T, \operatorname{dom}(T)) \in \mathbb{R}^{+} \cup\{+\infty\}$ satisfying

$(P 0) \Gamma^{m}$ is increasing, that is, $U$ extends $T$ implies that

$$
\Gamma^{m}(U, \operatorname{dom}(U)) \geq \Gamma^{m}(T, \operatorname{dom}(T)) .
$$

(P1) If $U$ has total domain satisfying $\Gamma^{m}\left(U, \mathbb{R}^{n}\right)<+\infty$, then the total function $u$ defined by $u(x):=U(x)(x)$ is in $C^{m}$ and $D^{m} u$ is Lipschitz.

(P2) If $u \in C^{m}\left(\mathbb{R}^{n}\right)$ with $D^{m} u$ Lipschitz, then

$$
\Gamma^{m}\left(U, \mathbb{R}^{n}\right)=\operatorname{Lip}\left(D^{m} u\right),
$$

where $U$ is the $m$-field associate to $u$.

(P3) For any $T$ such that $\Gamma^{m}(T, \operatorname{dom}(T))<+\infty, T$ extends to a total $m-$ field $U$ satisfying

$$
\Gamma^{m}\left(U, \mathbb{R}^{n}\right)=\Gamma^{m}(T, \operatorname{dom}(T)) .
$$

\subsection{Relationships between $\Gamma^{1}(F ; \Omega)$ and $\operatorname{Lip}(D f ; \Omega)$}

It is worth asking what is it the relationship between 1-field Lipschitz constant $\Gamma^{1}(F ; \Omega)$ and $\operatorname{Lip}(D f, \Omega):=\sup _{x \neq y \in \Omega} \frac{\|D f(x)-D f(y)\|}{\|x-y\|}$ ? The two are equal when $\Omega=\mathbb{R}^{n}$, but in general one only has $\operatorname{Lip}(D f, \Omega) \leq \Gamma^{1}(F, \Omega)$ and the strict inequality may happen even when $\Omega$ is convex (see $[20,21])$. It remains a very interesting question whenever the equality holds. It turns out that the answer depends heavily on the boundary of $\Omega$. In [21], Le Gruyer and Phan show that:

Theorem 4.3 Let $\Omega$ be an open set in $\mathbb{R}^{n}$. We have

$$
\Gamma^{1}(F ; \Omega)=\max \left\{\operatorname{Lip}(D f ; \Omega), \Gamma^{1}(F ; \partial \Omega)\right\} .
$$

Moreover, they prove that $\operatorname{Lip}(D f, \Omega)=\Gamma^{1}(F, \Omega)$ when either the supremum in the definition of $\Gamma^{1}(F, \Omega)$ is attained, or $\Gamma^{1}(F, \Omega)=\Gamma^{1}\left(F, \Omega^{\prime}\right)$ for some compact subset $\Omega^{\prime}$ of $\Omega$. 


\subsection{Extremal MLEs and AMLEs for 1-field}

Suppose that $\Gamma^{1}(F, \Omega)<+\infty$. From Theorem 4.1, there exists $g \in C^{1,1}\left(\mathbb{R}^{n}, \mathbb{R}\right)$ such that $\left.g\right|_{\Omega}=f,\left.\quad \nabla g\right|_{\Omega}=D f$ and the 1 -field $G$ associated to $(g, \nabla g)$ satisfies $\Gamma^{1}(F, \Omega)=\Gamma^{1}\left(G ; \mathbb{R}^{n}\right)$. Such a map $G$ is called a minimal Lipschitz extension (MLE) of $F$.

In 2015, Le Gruyer and Phan [21] constructed explicitly the sup-inf formulas for two extremal MLEs $U^{+}$and $U^{-}$, namely

$$
U^{-}(x)(x) \leq G(x)(x) \leq U^{+}(x)(x), \forall x \in \mathbb{R}^{n}
$$

for every MLE $G$ of $F$. Moreover, they proved that if $\Omega$ is finite, then $U^{ \pm}$are absolutely minimal Lipschitz extensions (AMLE) of $F$, namely

$$
\Gamma^{1}\left(U^{ \pm}, D\right)=\Gamma^{1}\left(U^{ \pm}, \partial D\right)
$$

for any open, bounded set $D$ satisfying $\bar{D} \subset \mathbb{R}^{n} \backslash \Omega$.

This result give the existence of AMLEs of $F$ when $\Omega$ is finite. In general, we have not uniqueness, since it may happen $u^{-}<u^{+}$. In fact, we may even have infinity AMLE of $F$.

Recently, in 2018, Azagra, Le Gruyer and Mudarra [6] provide necessary and sufficient conditions for the 1-field $F$ to admit an extension $G$ associated to $(g, \nabla g)$ with $g$ convex and of class $C^{1,1}$. As a consequence, they obtain a simple explicit formulas for $U^{+}$and $U^{-}$.

When $\Omega$ is infinite, $U^{+}$and $U^{-}$are extremal MLEs, but in general are not AMLE of $F$ (see [21]). The question on the existence of an AMLE for a 1-field when $\Omega$ is infinite remains a very difficult open problem.

Problem 4.4 Whether there exists an AMLE of 1-field F?

\section{References}

[1] Armstrong, S.N., Smart, C.K.: As easy proof of Jensen's theorem on the uniqueness of infinity harmonic functions. Calc. Var. Partial Differential Equation. 37(3-4), 381-384 (2010).

[2] Aronsson, G.: Minimization problems for the functional sup $p_{x} F\left(x, f(x), f^{\prime}(x)\right)$. Ark. Mat. 6, 33-53 (1965).

[3] Aronsson, G.: Minimization problems for the functional sup $p_{x} F\left(x, f(x), f^{\prime}(x)\right)$ II . Ark. Mat. 6, 409-431 (1966).

[4] Aronsson, G.: Extension of functions satisfying Lipschitz conditions. Ark. Mat. 6, 551561(1967).

[5] Aronsson, G.: On certain singular solutions of the partial differential equation $u_{x}^{2} u_{x x}+$ $2 u_{x} u_{y} u_{x y}+u_{y}^{2} u_{y y}=0$. Manuscripta Math. 47, no. 1-3, 133-151 (1984).

[6] Azagra, D., Le Gruyer, E., Mudarra, C.: Explicit formulas for $C^{1,1}$ and $C_{\text {conv }}^{1, \omega}$ extensions of 1-jets in Hilbert and superreflexive spaces Journal of Functional Analysis 274 (10), 3003-3032, (2018).

[7] Aronsson, G., Crandall, M.G, Juutinen, P.: A tour of the theory of Absolutely Minimizing Functions. Bull. Am. Math. Soc. 41(4), 439-505 (2004).

[8] Benyamini, Y., Lindenstrauss, J.: Geometric Nonlinear Functional Analysis, Vol 1. American Mathematical Society Colloquium Publications, vol 48. American Mathematical Society, Providence (2000). 
[9] Bezdek, K., Connelly, R. Pushing disks apart- the kneser-poulsen conjecture in the plane, J. reine angew. Math. 553, 221-236 (2002).

[10] Champion, T., Pascale, L.D. : Principles of comparison with distance functions for absolute minimizers, J. Convex Anal. 14, no. 3, 515-541 (2007).

[11] Crandall, M.G., Evans, L.C., Gariepy, R.F. : Optimal Lipschitz extensions and the infinity laplacian, Calc. Var. Partial Differential Equation. 13, no. 2, 123-139 (2001).

[12] Crandall, M.G., Lions, P.L.: Viscosity solutions of Hamilton-Jacobi equations, Trans. Amer. Math. Soc. 277, no. 1, 1-42 (1983).

[13] Evans, L.C. and Smart, C. K.: Everywhere differentiability of infinity harmonic functions , Calc. Var. 42, 289-299 (2011)

[14] Hirn, M.J., Le Gruyer, E.L.: A general theorem of existence of quasi absolutely minimal Lipschitz extensions. Math. Ann. 359(3), 595-628 (2014).

[15] Glaeser, G.: Études de quelques algèbres tayloriennes. J. d' Analyse math. 6, 1-124 (1958).

[16] Jensen, R.: Uniqueness of Lipschitz extension: minimizing the sup-norm of gradient. Arch. Rational Mech. Anal. 123(1), 57-74(1993).

[17] Juutinen, P.:, Absolutely minimizing Lipschitz extension on a metric space. Ann. Acad. Sci. Fenn. Math. 27, 57-67 (2002).

[18] Kirszbraun,M.D.: Über die zusammenziehende und Lipschitzsche Transformationen. Fund. Math. 22, 77-108 (1934).

[19] V.M. Kneser, Einige bemerkungen uber das minkowskische flachenmass, Archiv der Mathematik 6, 382-390 (1955).

[20] Le Gruyer, E.: Minimal Lipschitz extensions to differentiable functions defined on a Hilbert space. Geom. Funct. Anal. 19(4), 1101-1118 (2009).

[21] Le Gruyer, E., Phan, T.V. Sup-Inf explicit formulas for minimal Lipschitz extensions for 1-fields on $\mathbb{R}^{n}$. J. Math. Anal. Appl. 424(1-2), 1161-1185 (2015).

[22] McShane, E.J.: Extension of range of functions. Bull. Amer. Math. Soc. 40, 837-842 (1934).

[23] Peres, Y., Schramm, O., Sheffield, S., Wilson, D.B.: Tug-of-war and the infinity Laplacian. J. Am. Math. Soc. 22(1), 167-210 (2009).

[24] Poulsen, E.T. Problem 10, Mathematica Scandinavica 2 , 346 (1954).

[25] Savin, O.: $C^{1}$ regularity for infinity harmonic functions in two dimensions. Arch. Rational Mech. Anal. 176(3), 351-361 (2006).

[26] Schwartz, J. T. Nonlinear functional analysis, New York: Gordon and Breach Science (1969), 100-108.

[27] Sheffield, S., Smart, C.K .: Vector-valued optimal Lipschitz extension. Commun. Pure. Appl. Math. 65(1), 128-154 (2012).

[28] Valentine, F.A. On the extension of a vector function so as to preserve a Lipschitz condition, Bull. Am. Math. Soc. 49, 100-108 (1943).

[29] Whitney, H.: Analytic extensions of differentiable functions defined in closed sets. Trans. Amer. Math. Soc. 36, 63-89 (1934). 